\title{
AGDV 2015: Berlin, Bismarck und die Dermatologie
}

\author{
AGDV 2015: Berlin, Bismarck and Dermatology
}

Autoren

C. R. Löser ${ }^{1}$, M. Geiges ${ }^{2}$

Institute

${ }^{1}$ Hautklinik, Hauttumorzentrum, Klinikum Ludwigshafen

${ }^{2}$ Moulagenmuseum Universität Zürich und Hautarztpraxis,

Kloten/Schweiz
Für 10 Jahre war Dresden der Veranstaltungsort für die Kongresse der Deutschen Dermatologischen Gesellschaft (DDG). In diesem Jahr fand die 48. Tagung erstmals wieder in Berlin statt und damit auch das Arbeitstreffen der Arbeitsgemeinschaft für Geschichte der Dermatologie und Venerologie (AGDV). Satzungsgemäß gab es eine ordentliche Mitgliederversammlung. Dr. Michael Geiges aus Zürich gab seinen Vorsitz ab, um sich verstärkt europäischen Aufgaben als Präsident der ESHDV (European Society for the History of Dermatovenerology) zu widmen. Die AGDV dankt ihm sehr für sein Engagement und seine bedachte Führung. Er hatte den Vorsitz seit 2009 inne und bleibt dem Vorstand der AGDV erfreulicherweise als Beisitzer erhalten.

Aus der wissenschaftlichen Sitzung im offiziellen Programm der DDG werden einige Beiträge in dieser Ausgabe der Aktuellen Dermatologie veröffentlicht. Die ausgewählten Arbeiten haben jeweils in besonderer Weise ihren Bezug zum Veranstaltungsort Berlin: Dr. Andreas Mettenleiter aus Würzburg präsentiert uns eine Spurensuche zum Dermatologen Kurt Boas, der nach einer Inhaftierung im KZ Sachsenburg möglicherweise dort ermordet wurde; oder war er 1937 in Berlin als niedergelassener Hautarzt tätig? Den bewegenden Lebenslauf des berühmten Dermatologen Abraham Buschke, der das Rudolf-Virchow-Krankenhaus in Berlin leitete, untersucht Dr. Martin Lorenz aus Kaiserslautern.

Zum 200. Geburtstag des Reichsgründers Otto von Bismarck (1815-1898) passen zwei weitere Beiträge. Theodor Althoff wurde auch als „Bismarck des deutschen Universitätswesens“ bezeichnet. Sein Wirken als Wissenschaftsorganisator wird im Beitrag von Dr. Christoph Löser aus Ludwigshafen skizziert. Und die Biografie von Ernst Schweninger, dem Leibarzt Otto von Bismarcks, stammt vom wichtigsten Vorstandsmitglied, Herrn Dr. Volker Wendt aus Westerstede Schatzmeister der AGDV.

Der Dank gebührt allen Rednern und Autoren für ihre Beiträge sowie Frau Professor Bayerl und dem Thieme Verlag für die immer großzügige Unterstützung bei der Veröffentlichung. Ein ganz besonderer Dank gilt dem Sekretär der AGDV, Herrn Dr. Martin Lorenz, der seit 2014, unterstützt von seiner Frau Tina, selbst Dermatologin und ebenfalls historisch engagiert, die Erneuerung der Gesellschaft aktiv unterstützt und alle Beiträge zusammengetragen hat.

Nach der Arbeitssitzung gab es für alle Interessierten noch eine Exkursion in das Berliner Medizinhistorische Museum der Charité. Geführt vom bekannten Moulagenforscher und Instituts-Direktor Prof. Dr. Thomas Schnalke, besuchten wir die sehenswerte Hörsaalruine, die Sammlung von Rudolf Virchow sowie die aktuelle Ausstellung „Arsen und Spitzenforschung“ zu Leben und Arbeit von Paul Ehrlich ( $\bullet$ Abb. 1).

Nach der Tagung ist vor der Tagung und so hoffen wir, dass der ein oder andere beim Lesen der Beiträge motiviert wird, zum nächsten Arbeitstreffen der AGDV bei der Fortbildungswoche nach München zu kommen. Am Dienstag, den 26.7.2016 verleiht die AGDV im Rahmen ihrer Sitzung außerdem die Ehrenmitgliedschaft an Herrn Prof. Dr. Dr. h.c. mult. Thomas Ruzicka und Herrn Prof. Dr. Dr. Johannes Ring. Wir freuen uns schon jetzt auf Ihre Teilnahme.

\section{Dr. Christoph R. Löser \\ Vorsitzender der AGDV \\ Dr. Michael Geiges \\ Präsident der ESHDV}

Bibliografie

DOI http://dx.doi.org/ 10.1055/s-0041-109088 Akt Dermatol 2015; 41: 501 (c) Georg Thieme Verlag KG Stuttgart · New York ISSN 0340-2541

\section{Korrespondenzadresse}

\section{Dr. Christoph R. Löser}

Hautklinik, Hauttumorzentrum Klinikum Ludwigshafen Bremserstr. 79 67063 Ludwigshafen loeserc@klilu.de

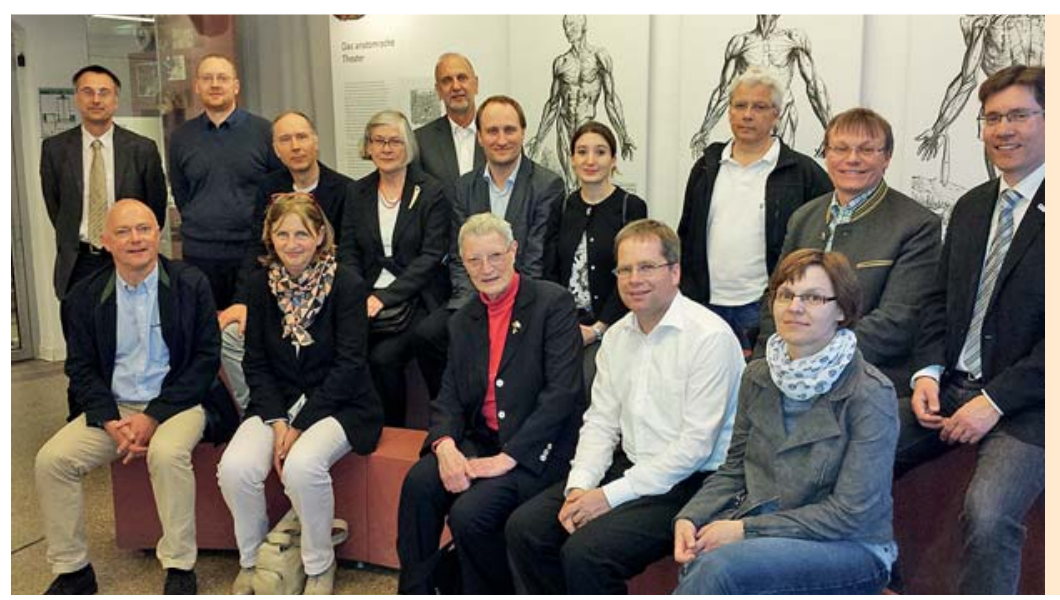

Abb. 1 Prof. Thomas Schnalke (zweite Reihe, Erster von links) und Mitglieder der AGDV im Medizinhistorischen Museum der Charité Berlin am 29.4.2015 (Foto: Prof. Dr. Sonja Ständer). 